\title{
TERMINAL DE ÓMNIBUS DE LAS BREÑAS [CHACO]
}

I IRIS CAROLINA ALMANZA/ MARÍA DEL HUERTO MARTÍNEZ QUIROGA/ GONZALO CABÁS/ ALBANO MUCCHIELLI

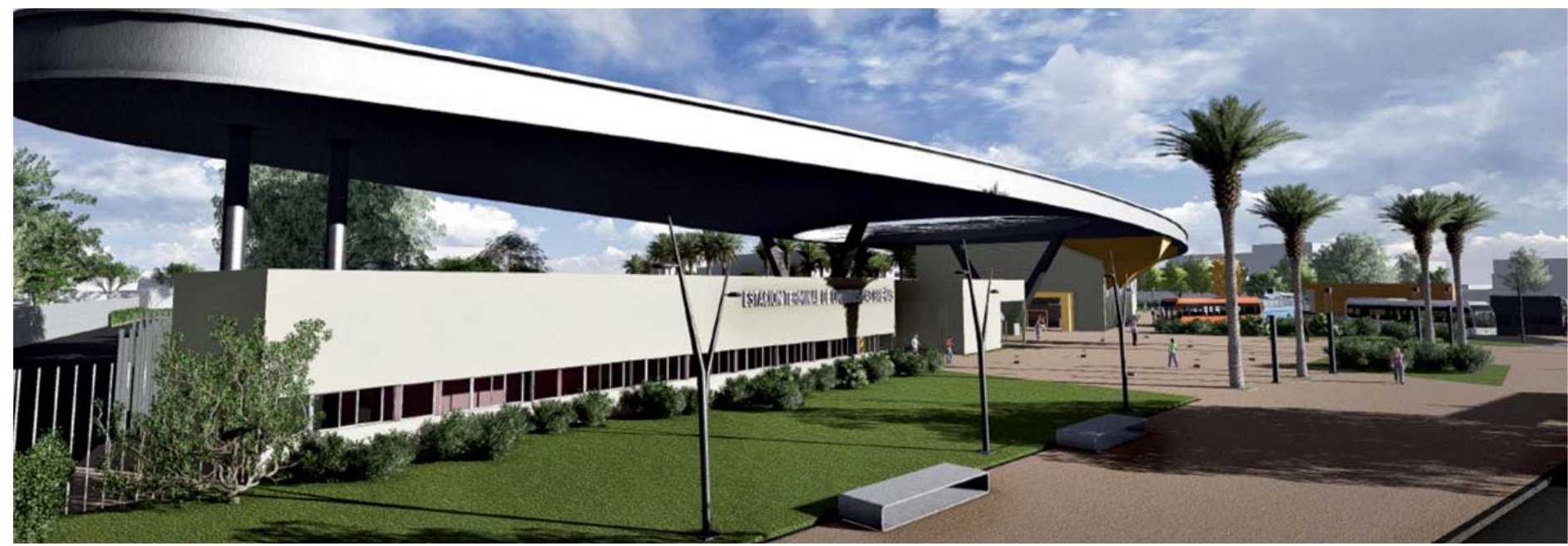




\section{RESUMEN}

El presente artículo tiene por objetivo exponer los fundamentos y decisiones de diseño de la propuesta del Concurso Provincial de Anteproyecto de la Terminal de Ómnibus de Las Breñas (Chaco), del cual se obtuvo el Primer Premio. Este concurso fue organizado por la Sociedad de Arquitectos del Chaco, y promovido por la Municipalidad de la Ciudad de Las Breñas, del Departamento 9 de Julio, de la provincia del Chaco.

\section{ABSTRACT}

This article aims to explain the fundaments and design decisions of the proposed winner of the first prize in the "Provincial Draft Contest Bus Terminal Las Breñas (Chaco)". This competition was organized by the Society of Architects of Chaco, and promoted by the Municipality of Las Breñas, in the province of Chaco.

IRIS CAROLINA ALMANZA. Arquitecta egresada de la Universidad Nacional del Nordeste. Socia fundadora de Grupo Mas Arquitectura, joven estudio de arquitectura dedicado a desarrollar proyectos, dirección de obra y diseño de interiores. Participó en el Concurso de Anteproyecto para el Museo Provincial de Arte Contemporáneo para la ciudad de Mar del Plata (2009). Coautora del 1.er premio del concurso Anteproyecto de Restauración, Refuncionalización y Puesta en Valor del Mercado Municipal de la provincia del Chaco (2012).

MARÍA DEL HUERTO MARTíNEZ QUIROGA. Arquitecta egresada de la Universidad Nacional del Nordeste. Socia fundadora de Grupo Mas Arquitectura, joven estudio de arquitectura dedicado a desarrollar proyectos, dirección de obra y diseño de interiores. Participó en el Concurso de Anteproyecto para el Museo Provincial de Arte Contemporáneo para la ciudad de Mar del Plata (2009). Coautora del 1.er premio del concurso Anteproyecto de Restauración, Refuncionalización y Puesta en Valor del Mercado Municipal de la provincia del Chaco (2012).

GONZALO CABÁS. Arquitecto egresado de la Universidad Nacional del Nordeste. Trabajó para la firma A\&D en departamento de Diseño y Asistencia en Obra (2002 a 2009). Socio gerente de Estudio LIBA, Identidad Global, Diseño Gráfico, Marketing, Arquitectura y Ejecución de Obras.

ALBANO MUCCHIELLI. Arquitecto egresado de la Universidad Nacional del Nordeste. Director creativo en LIBA, Estudio de Identidad Global, Diseño Gráfico, Marketing, Arquitectura y Ejecución de Obras. Desarrollador de marcas y generador creativo de proyecto. Asesor junto a Gonzalo Cabás de nuevas empresas en la implementación, gestión y desarrollo de proyectos. 


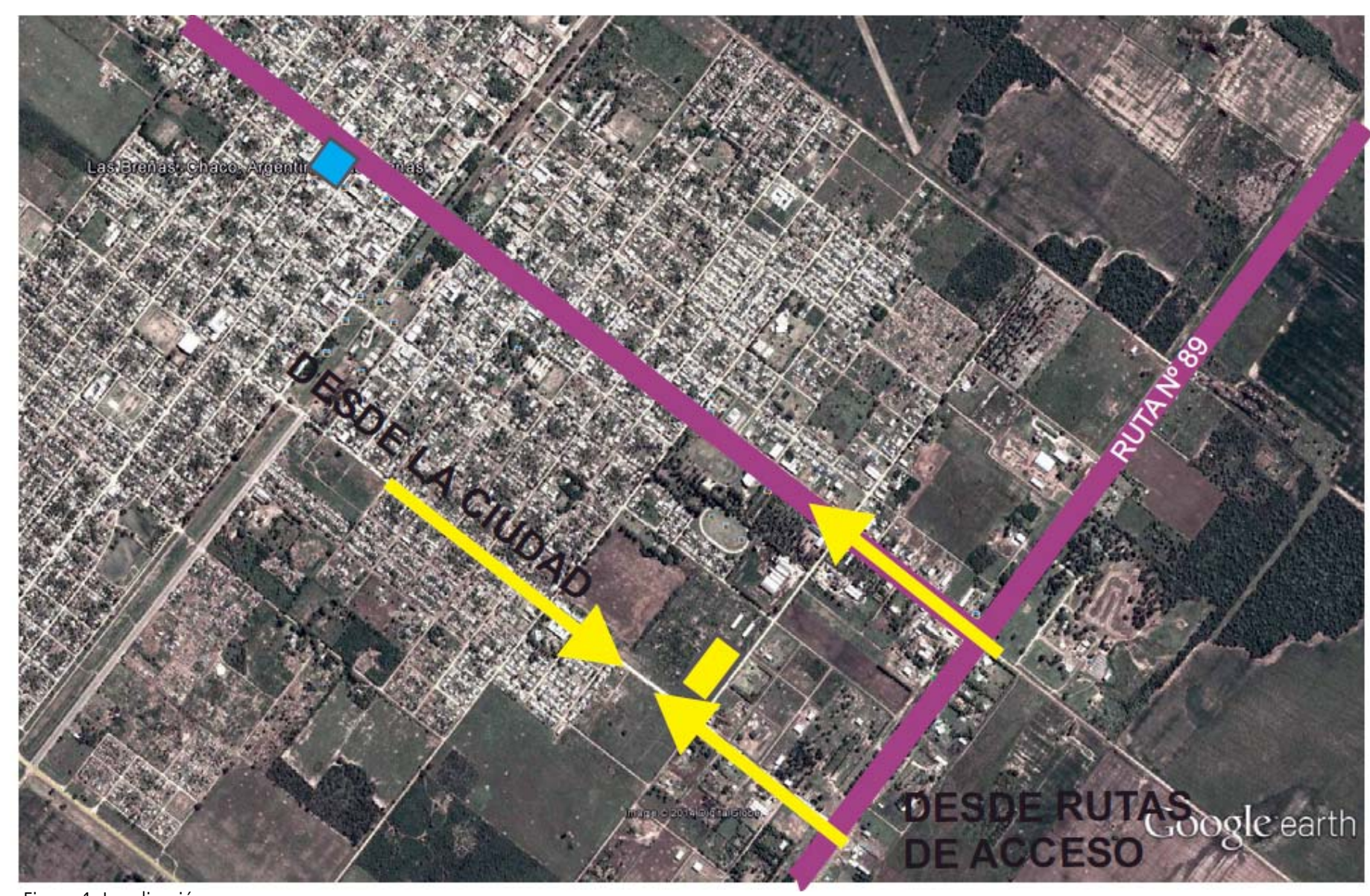

Figura 1. Localización

\section{LOCALIZACIÓN}

Conocida como la "Capital Provincial del Inmigrante", la ciudad de Las Breñas se encuentra situada geográficamente al sudoeste de la provincia del Chaco. Es la ciudad cabecera del Departamento 9 de Julio. Se encuentra articulada al territorio provincial a través de la ruta nacional $\mathrm{N} .{ }^{\circ} 16$, siendo su acceso la ruta provincial N. ${ }^{\circ} 89$.

El predio donde se emplazará la obra cuenta con $25.040 \mathrm{~m} 2$, y se encuentra ubicado entre las calles Enrique Freidenreij, Inmigrantes Sirios Libaneses y República Federativa del Brasil.

\section{CIRCULACIONES GENERALES}

Se propuso que la calle República de Brasil se transforme en una avenida de doble mano, y que en el resto de las circulaciones que llegan al predio se organice cada calle con un sentido único de circulación para que la entrada y salida de ómnibus no genere congestionamiento en el tráfico vehicular. La avenida Enrique Freidenreij será la arteria principal de comunicación entre la cuidad y la terminal y entre la terminal y los arribos y partidas de los ómnibus (figuras 1 y 2).

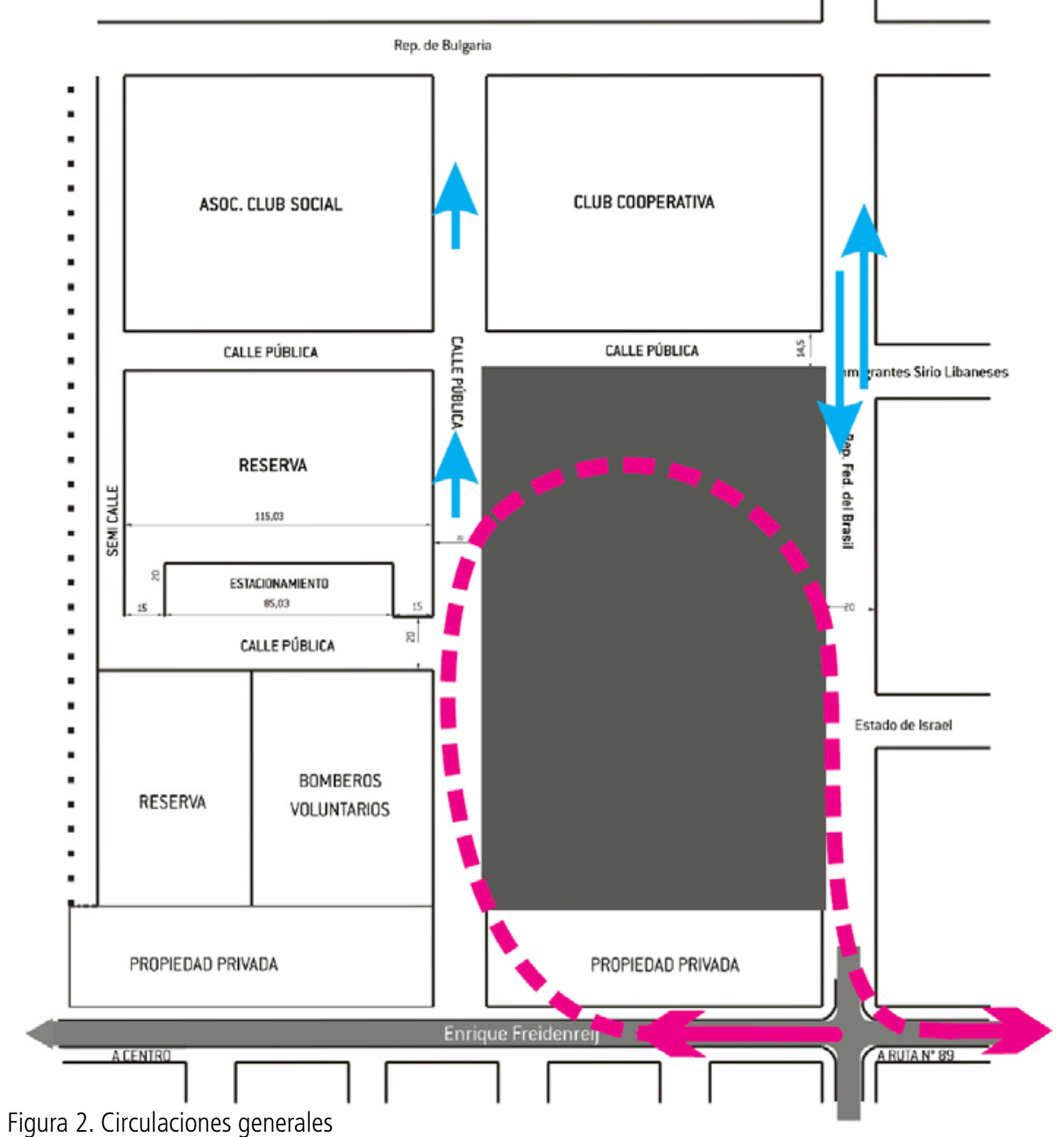

Figura 2. Circulaciones generales 


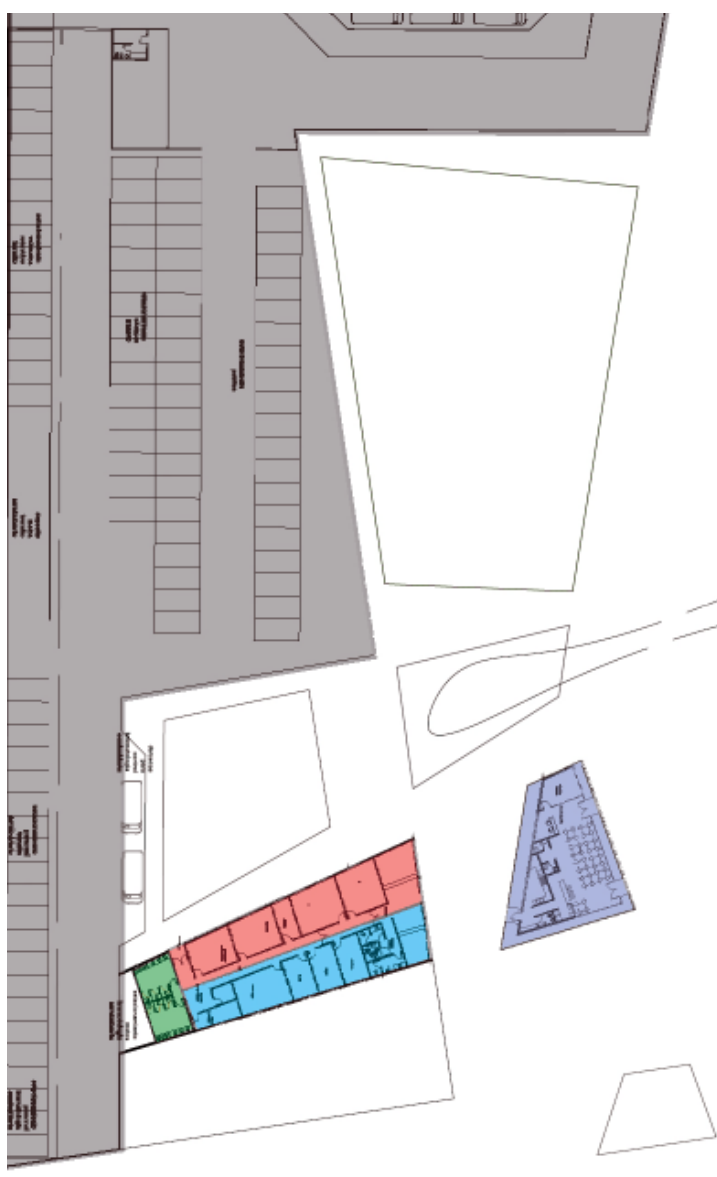

Figura 3. [propuesta de sectorización de actividades]

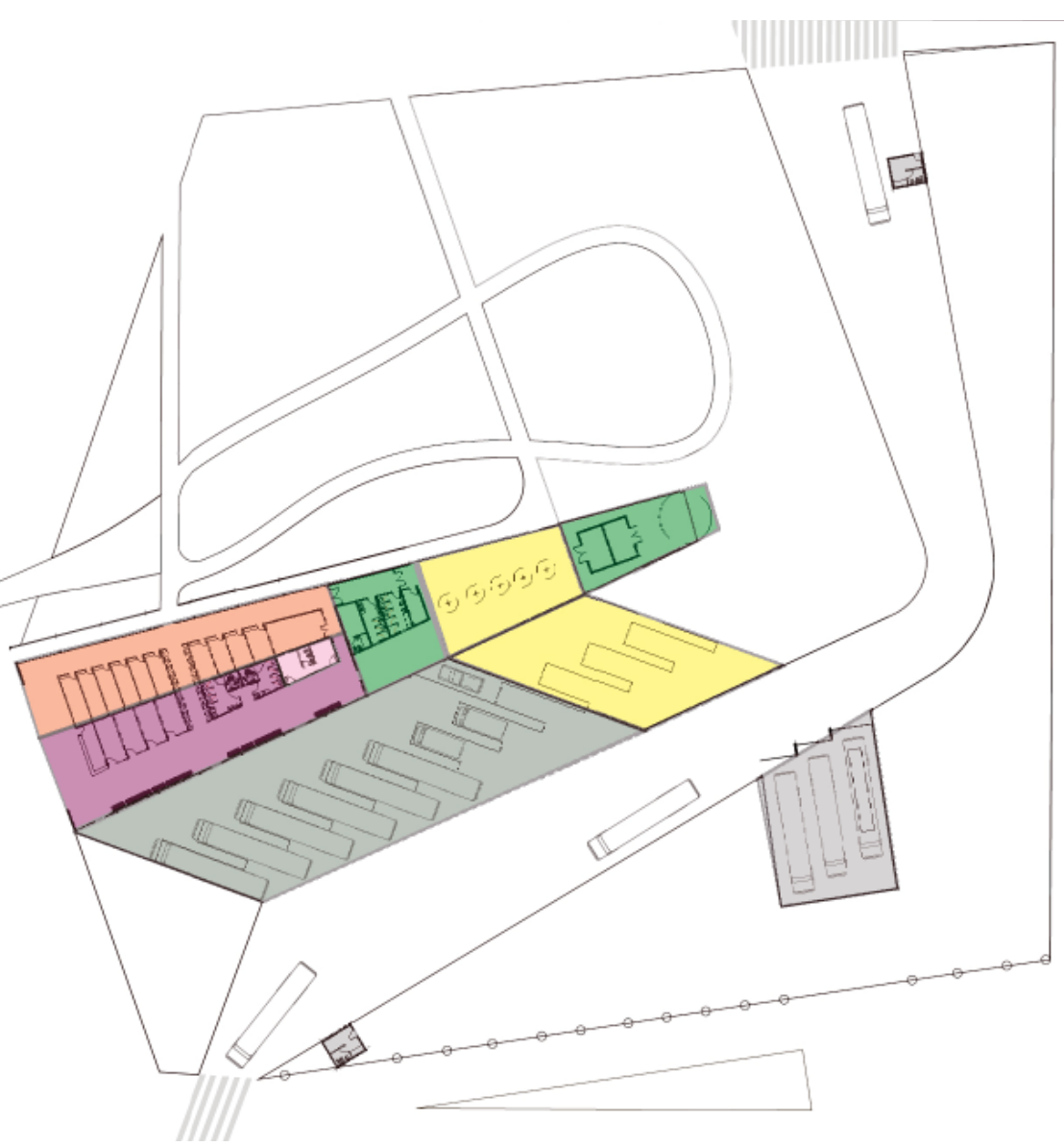

Figura 4. [Funcionalidad]

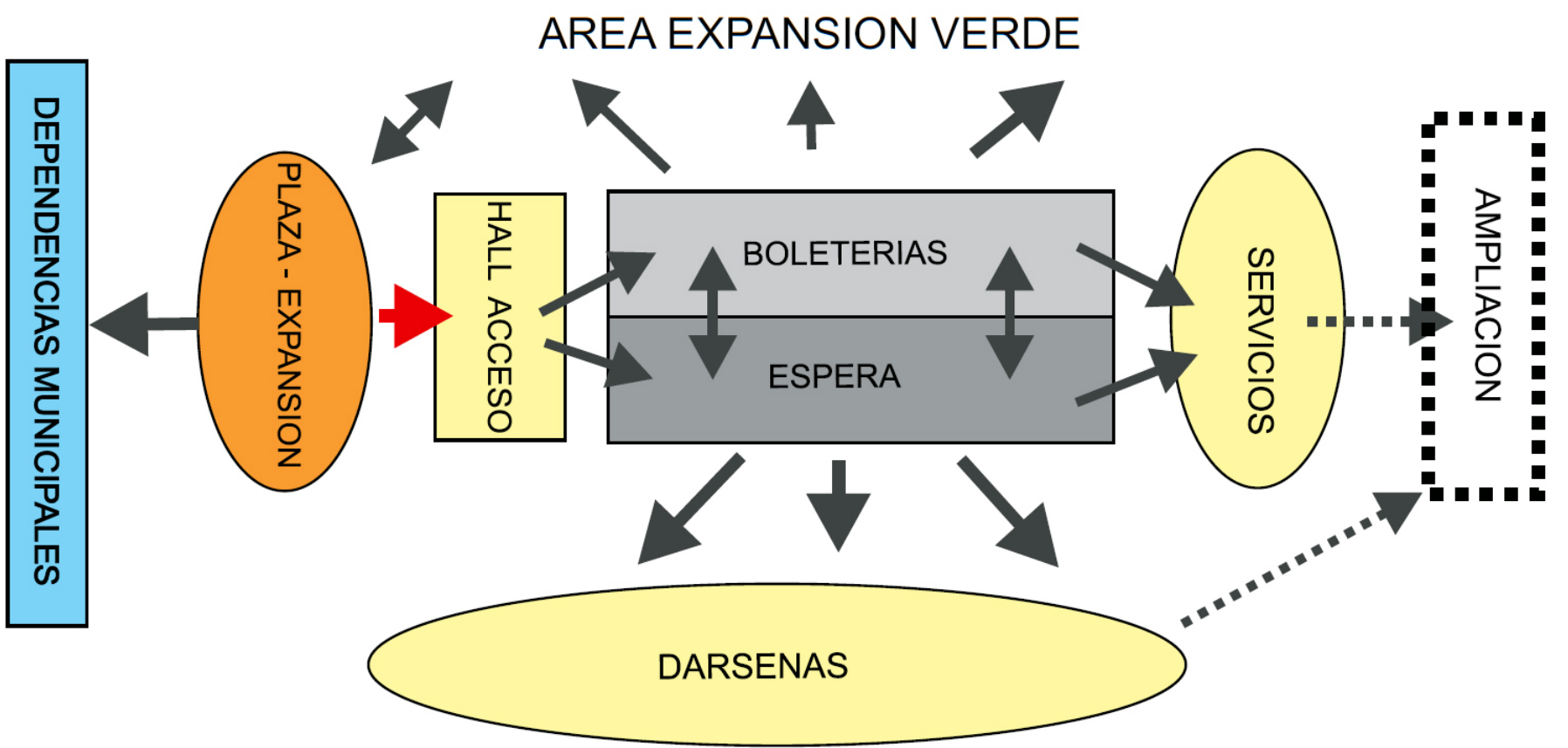

\section{FUNCIONALIDAD}

La propuesta funcional del edificio parte de la sectorización de las actividades, que da por resultado una gran isla longitudinal abierta hacia las circulaciones, halls, zonas de espera y áreas de apoyo. Esta isla está dividida en su eje longitudinal y aloja a las actividades comerciales, las salas de espera cubierta y semicubierta vinculadas directamente con las dársenas, para el mejor aprovechamiento del asoleamiento y de los vientos del sureste.
En otro de los sectores, ubicado sobre la fachada noroeste, se encuentran las boleterías y los depósitos de equipaje y encomiendas. En uno de los extremos del edificio se ubicaron el acceso principal y el hall, y en el otro extremo, las áreas de servicios, directamente relacionadas con el área técnica de la terminal (sala de máquinas, talleres mecánicos, etc.) y los accesos de ómnibus y de servicios.El acceso principal al edificio se da a través de la plaza central. Se propusieron además dos accesos secundarios, uno desde el corredor verde directo a las boleterías y el otro desde las dársenas (Figuras 3 y 4). 

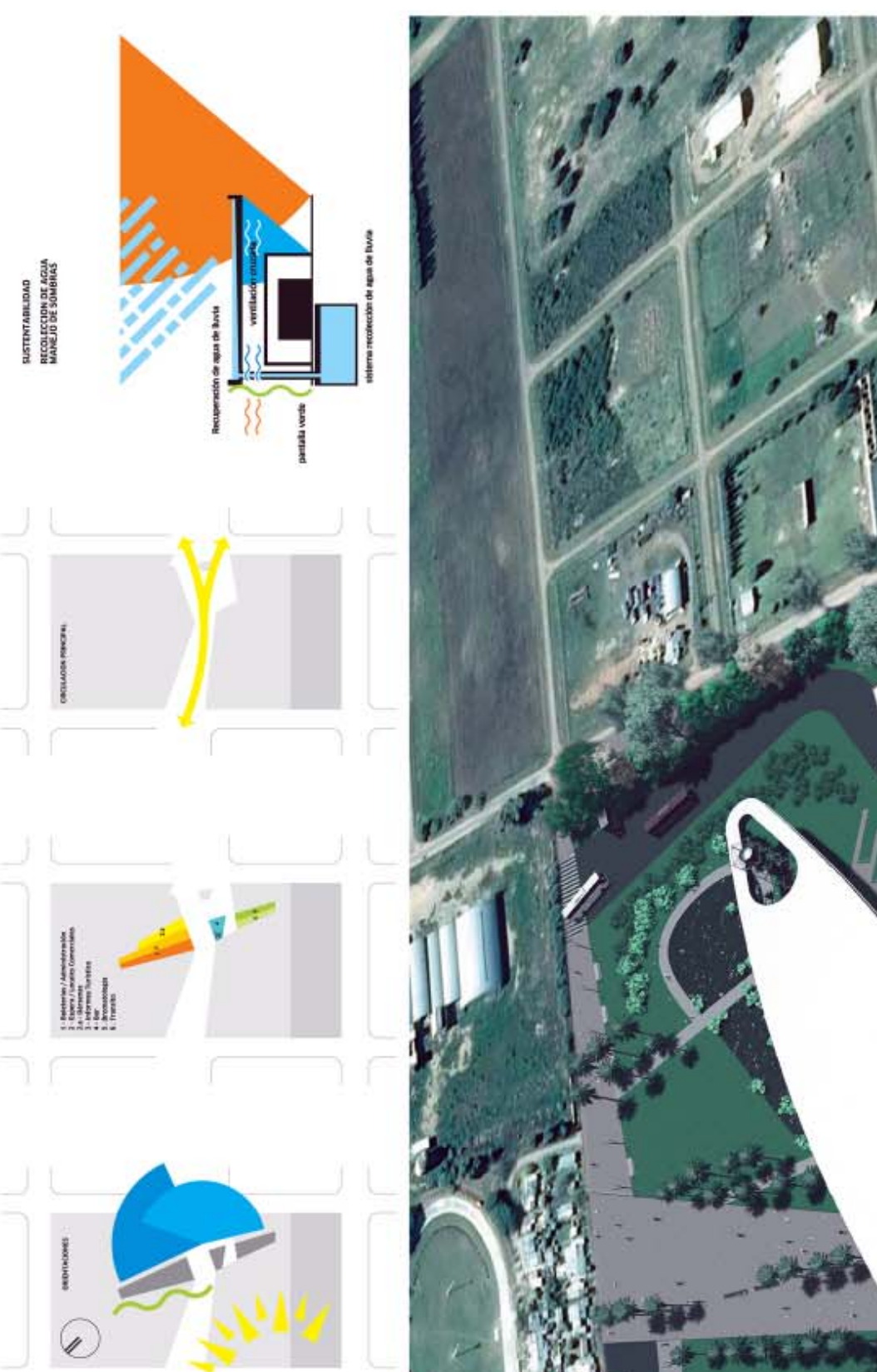

- III
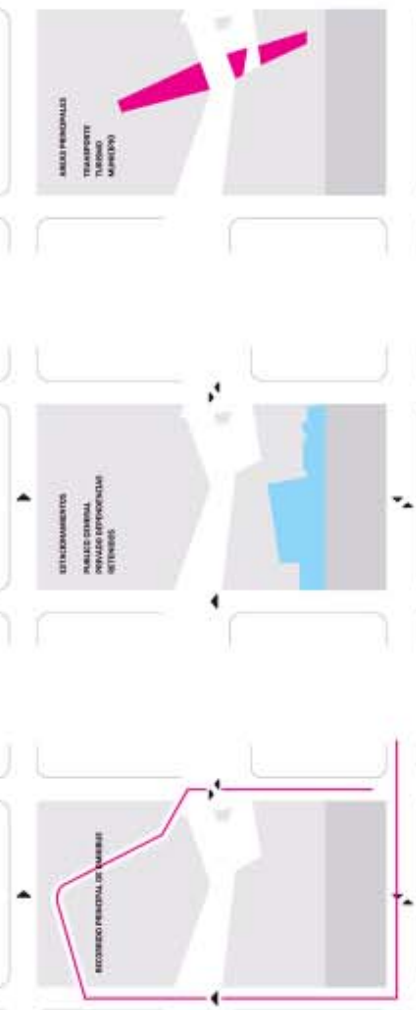

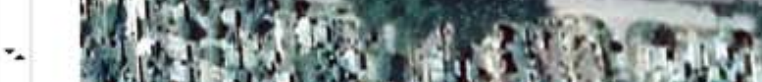
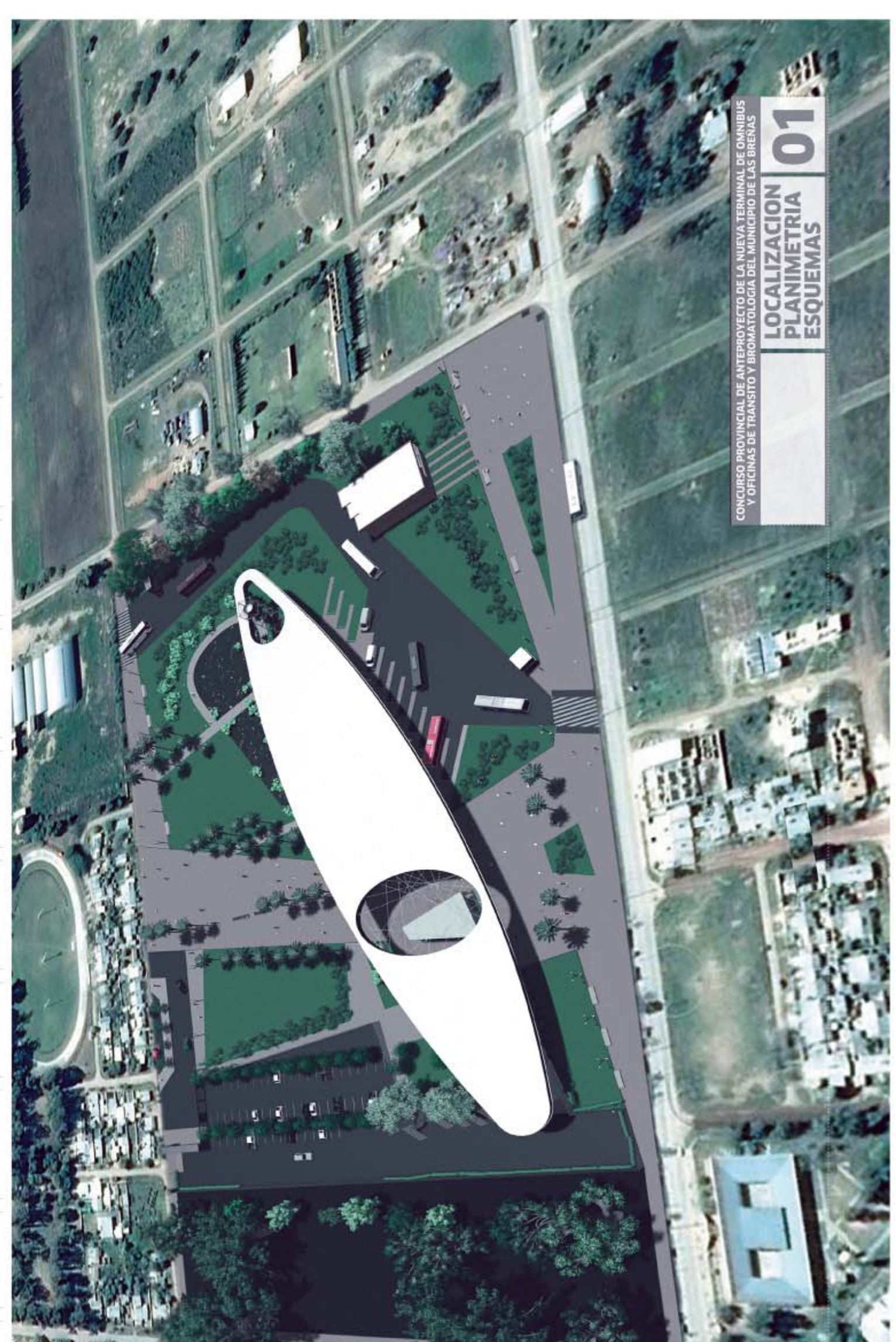



TERM I N A L

(1) rall De acceso

(2) BOLTERIIS

(1) INFOQMercion

(1) COMERCIOS

(ㄱ) DEPOSITO EQUNPANES

(c) SANITARIOS PUBLICOS

(7) OFICINAS ADMIIISTRATIVAS

(c) DEROSTIO ENCOMIENDA

(1) HärTACION DESCANSO CHOFER

(20) SANITARUOS PERSONAL

(11) DEPOSTIO MANTENIMIESTO

(9) SALA DE ESPERA

(3) ANDENES

(9) MLZZA SECA - FUTURA AMPLIACION

(19) DRQSENA DE SERVICIO

(18) AMPLucion DARSERus

(14) SALA DE GRUPO ERECTROGENO

(18) SALL Magevinas - CISTERNA

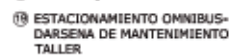

(2) CABISA DE CONTROL

(2) TURISMO Y CURTURA

(2) BAR - CAFtERLA

BROMATOLOGIA

(2) ATENCION AL PUBuCO

(23) OFCCINA DE INSPECCCION

(2) ARCHIVO

(2) DePOSITO RPOOUCTOS

(2) LBBORATORIO

23. ACCESO DE SERVICIO

(2) SanvirTRIOS - vestuarios

(2) OFCCE - UMPEZZA

T R A N S I T O

220 ATENCION AL PUELCO

(2) OFCCE- LMPEZZA

(9) Defostro MaNTENIMENTO

(19) SALON DE CAPMCTACIONES

(9) ofcimas pervadas

(9) ADMIINESTRACION

(29) SANITRARIOS PUBLCOSOS

ESTACIONAMIENTOS

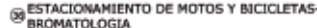

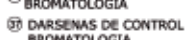

(29) ESTACIONAMIENTO PUBLCOO

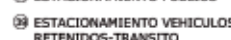

(79) DEPOSITO DE MOTOS-TRANSITO

(1) ESTACIONAMIENTO PERSONALL

(3) ESTACIONAMIENTO PERSONAL
BROMATOLOGIA

(3) ESTACIONMAMIENTO OFICIAL

(9) PAREDA TAXIS Y REMISES

(3) Preaph De COLECTIVOS 
Terminal de ómnibus de Las Breñas (Chaco)
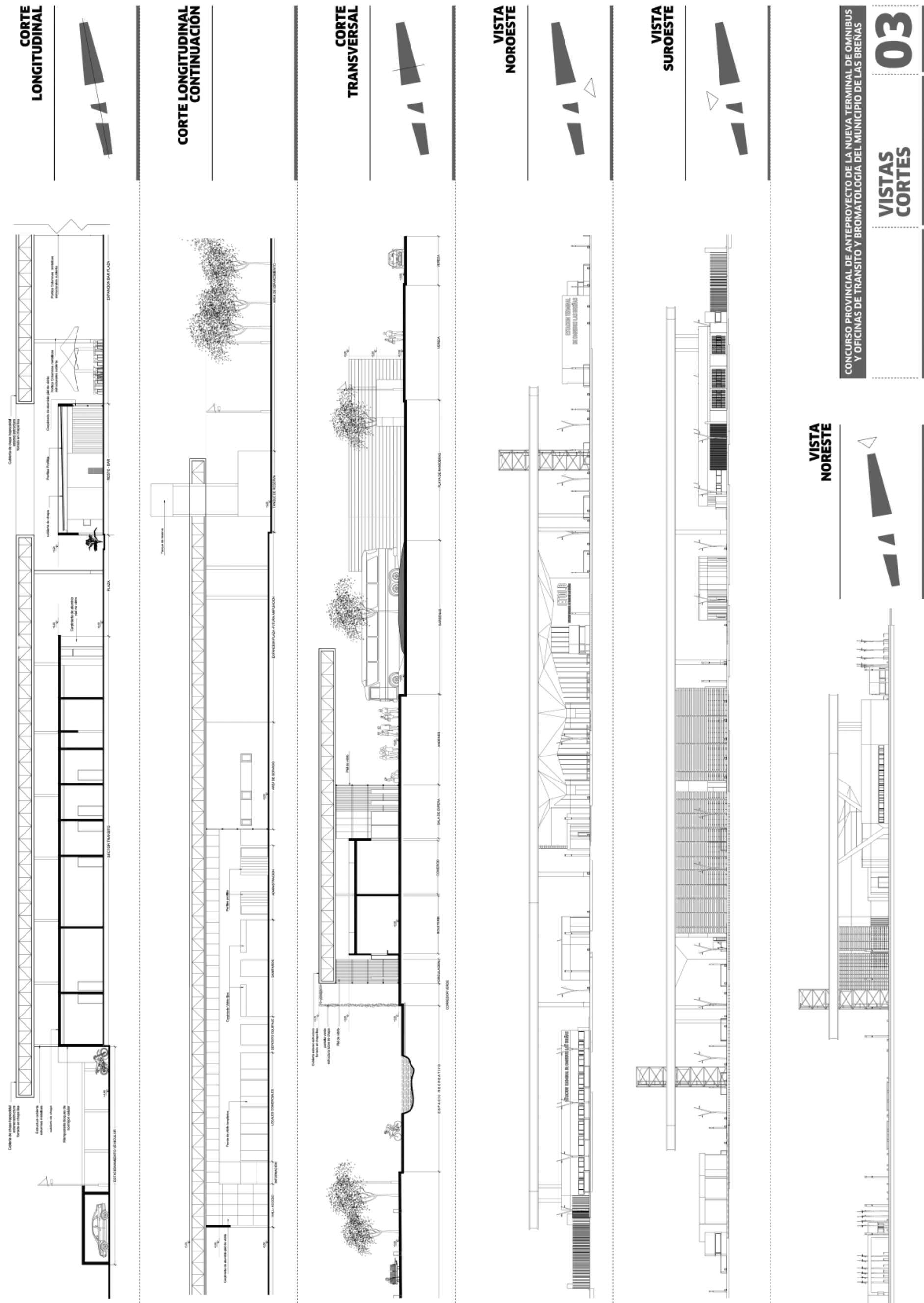

ADNea Revista de Arquitectura y Diseño del nordeste argentino- Vol 2 N. 2 (Septiembre 2014) Pp. 7-20 -ISSN 2347- 064X

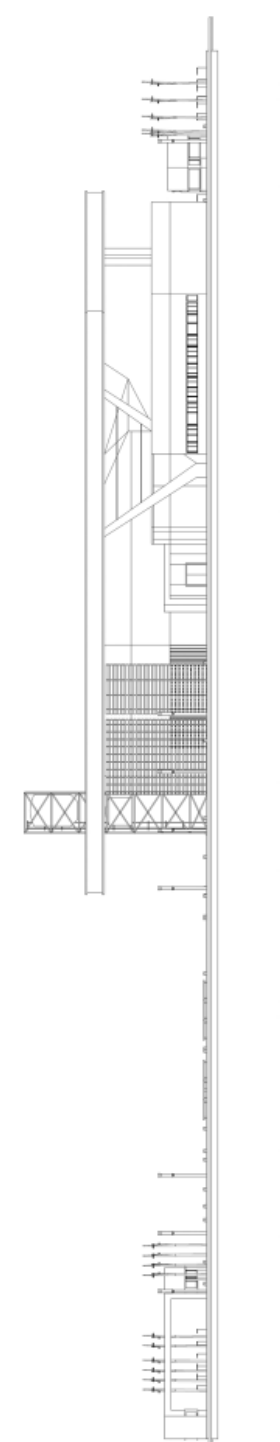



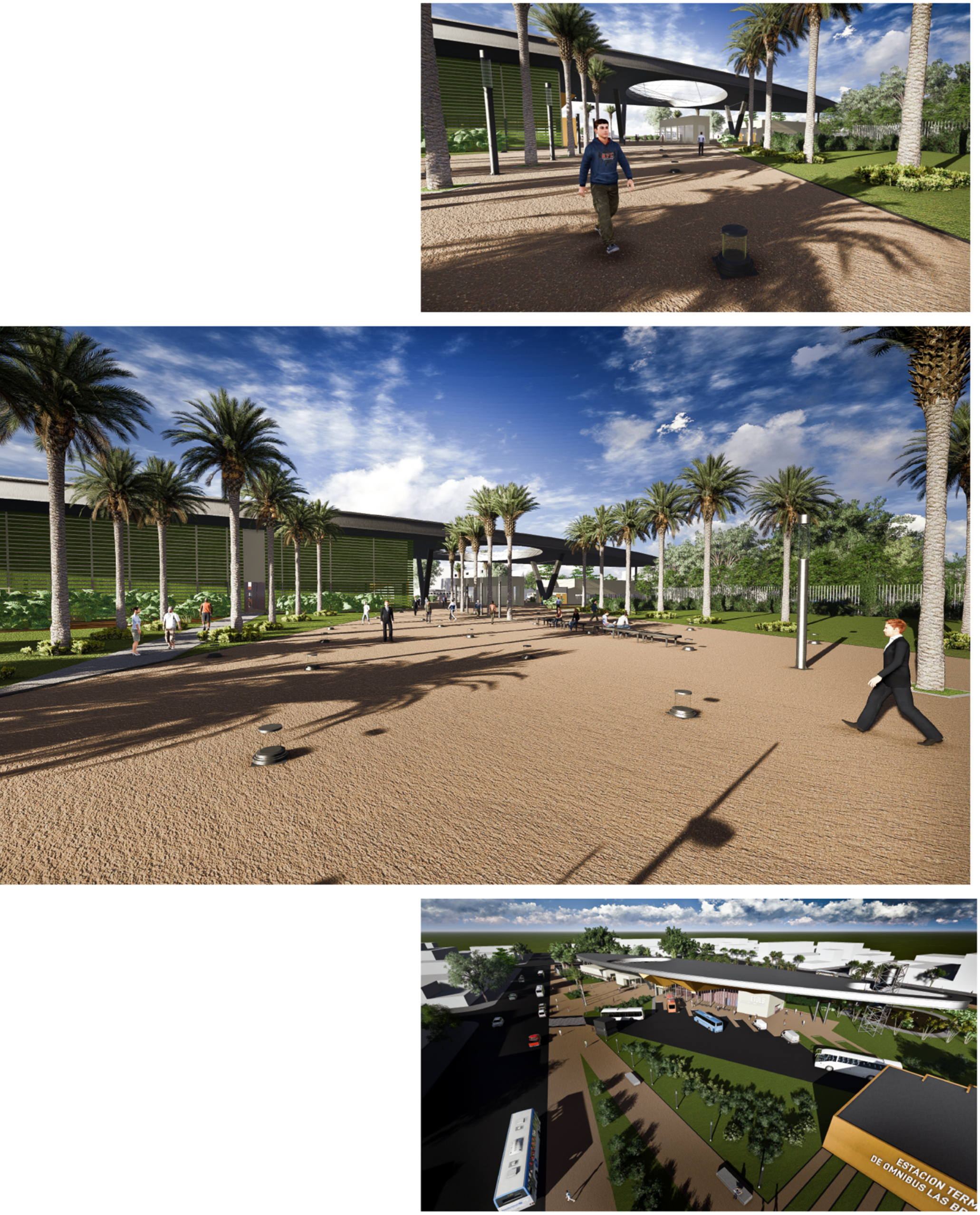

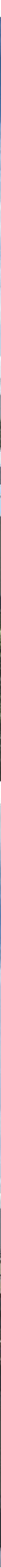

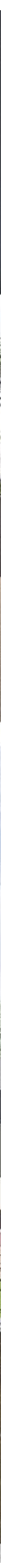




18.
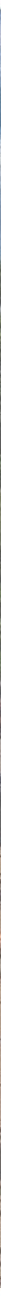


\section{EMPLAZAMIENTO}

Como resultado de condicionantes externos, tales como el clima y la interrelación con la trama urbana, y pensando en el desarrollo futuro del sector, el partido de la propuesta fue concebido como de modo abierto, organizado en tres bloques bien diferenciados, vinculados por un núcleo de circulación central. Se conformaron dos ejes, uno de oeste a este materializado a través de una plaza que se abre hacia las calles perimetrales, y el otro de norte a sur, donde se emplazaron los tres bloques.

El bloque principal aloja todas las actividades de la terminal y contempla su crecimiento. El bloque central nuclea las actividades de bares y oficina de turismo; conforma un hito dentro de la plaza, como un espacio permeable para todas las actividades del predio de toda la ciudad. El último bloque aloja las actividades de dirección de Tránsito y Bromatología, con sus áreas de servicio, control y estacionamientos (figura 5).

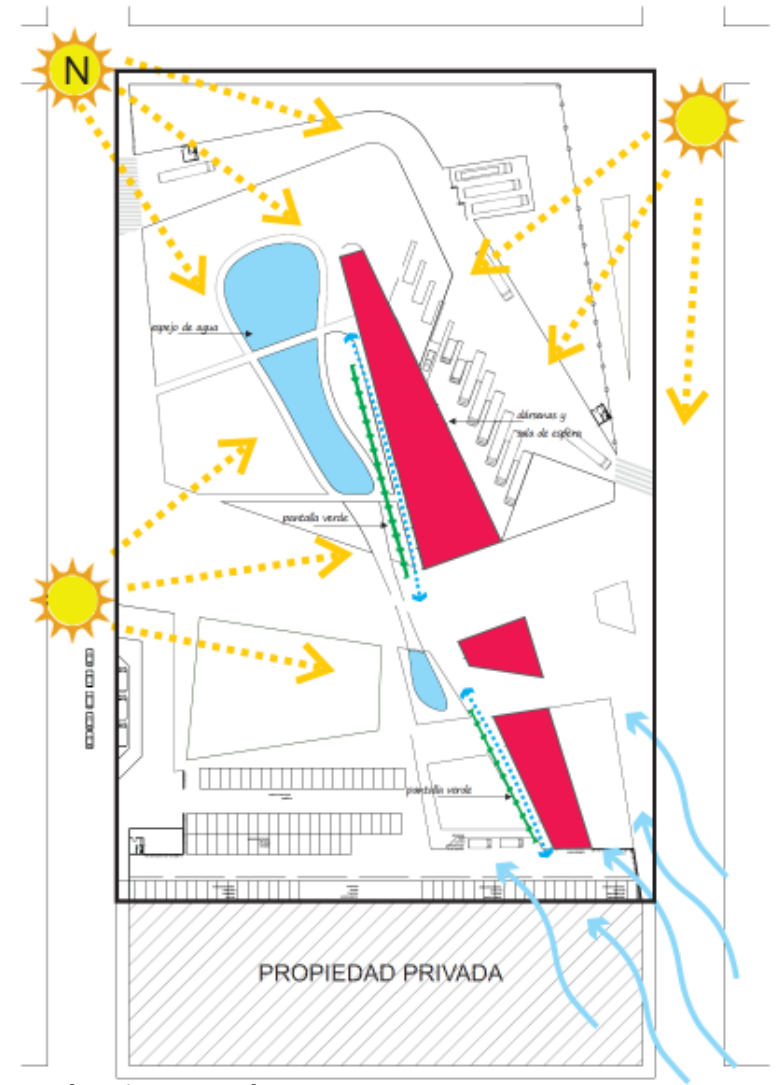

Figura 5. [Emplazamiento]

\section{CIRCULACIONES}

- Circulación de ómnibus: se realizará de manera transversal al predio, mediante la creación de un acceso sobre la futura calle proyectada y de una salida sobre la futura avenida República de Brasil, para evitar posibles congestiones de tránsito sobre esta avenida. Se propuso la circulación y playa de maniobra de los ómnibus sobre el extremo nordeste del predio, en áreas bien diferenciadas para el desarrollo de las distintas actividades y sin interferir con ellas.

-Circulación vehicular: los estacionamientos y las dársenas para taxis se ubicaron al suroeste del predio, para aprovechar esa fachada, que es poco favorecida por su orientación y poca visualización debido al predio lindero.

-Circulación peatonal: se diferenciaron las circulaciones principales que llevan a los distintos bloques de las secundarias y de las de servicios.

En las veredas se direccionan las circulaciones para invitar al peatón a un paseo urbano que concluye en la gran plaza central de acceso a los distintos edificios.

La propuesta ofrece senderos de esparcimientos y recreación que integran al predio con la ciudad.

\section{ORIENTACIONES Y SUSTENTABILIDAD}

La pauta primordial de la disposición de los bloques fue orientarlos casi completamente hacia el este-sureste, con la finalidad de lograr la mejor orientación, principalmente para el sector de las dársenas y sector de espera de la terminal. Por otro lado, se protegieron los bloques de las orientaciones más desfavorables con una pantalla verde, que atajará las radiaciones solares, tamizará la iluminación natural y, por estar separada del edificio, generará una corriente de aire que aislará el calor y dará mejor acondicionamiento térmico en el interior.

La gran altura de la cubierta en el bloque de la terminal permite que sea una sobrecubierta para los bloques del bar y las oficinas de turismo, tránsito y bromatología, que brinda una gran aislación a las altas temperaturas y al aire caliente que 


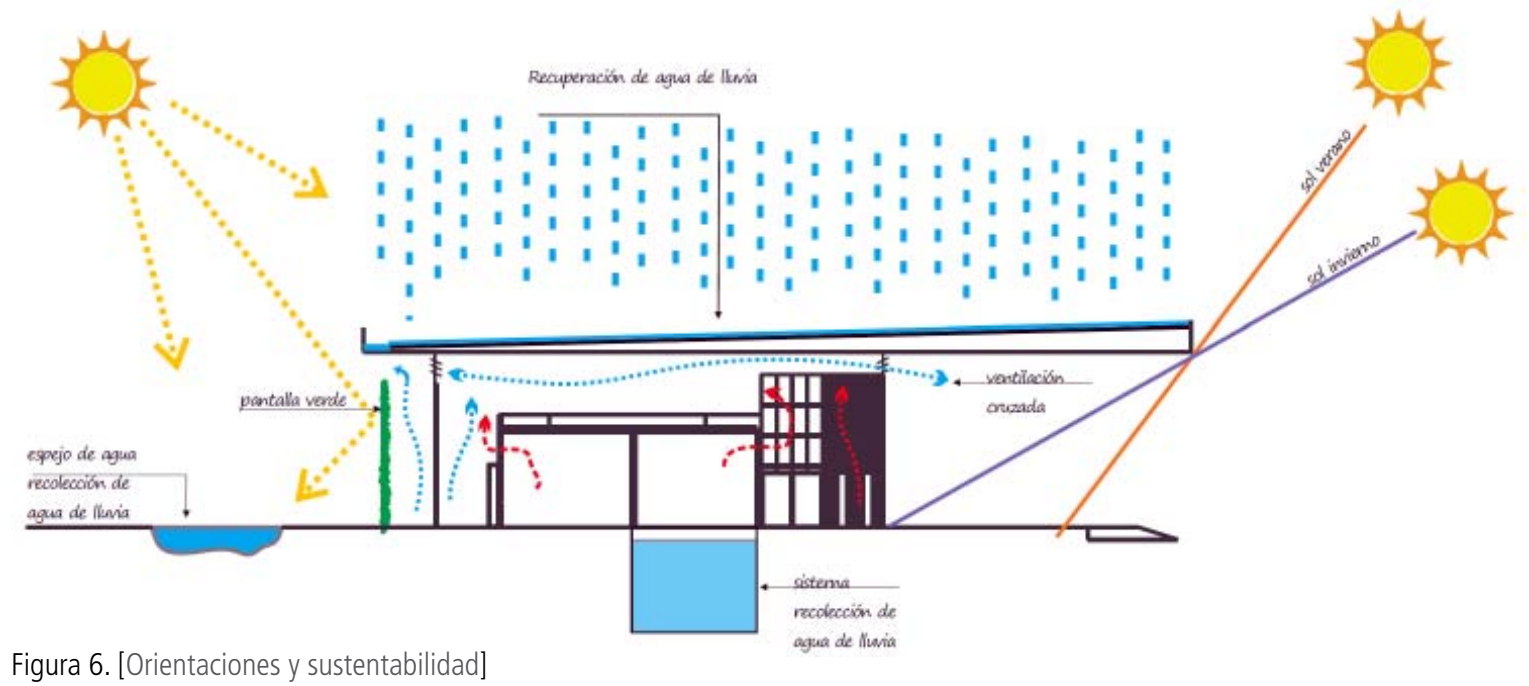

Figura 6. [Orientaciones y sustentabilidad]

MATERIALIZACIÓN recolección del agua de lluvia, la cual se almacena en un tanque, y se la utiliza para canillas de servicio, descarga de inodoros y duchas.

La propuesta contempla también un espejo de agua, que tiene tres finalidades: por un lado ayudará a reducir la incidencia del calor sobre el edificio; por otro lado, será un recurso que contribuirá al desarrollo de actividades recreativas y de ocio de la ciudad y, por último, será un reservorio de agua de lluvia, para ser utilizada para el riego de las áreas verdes del predio.

Se contemplaron en todos los espacios de la isla central de la terminal grandes ventanas, que dejarán entrar la iluminación natural durante el día para disminuir el consumo de energía (figura 6).
El sistema constructivo del edificio es tradicional, fácil de construir con materiales y mano de obra local. La mampostería portante es de bloque de hormigón celular; las cubiertas, de chapa galvanizada trapezoidal, con correas metálicas para las cubiertas del bloque de las oficinas de tránsito, bromatología, turismo y el bar.

La gran cubierta que abarca los tres bloques se sostiene con una estructura metálica compuesta por columnas, vigas reticuladas y una estéreo-estructura para cubrir grandes luces.

Las carpinterías son de aluminio, y para los grandes paños vidriados se propuso una piel de vidrio con un cristal "stop sol". Para los solados interior y exterior se propuso una combinación de materiales de la zona (figura 7).

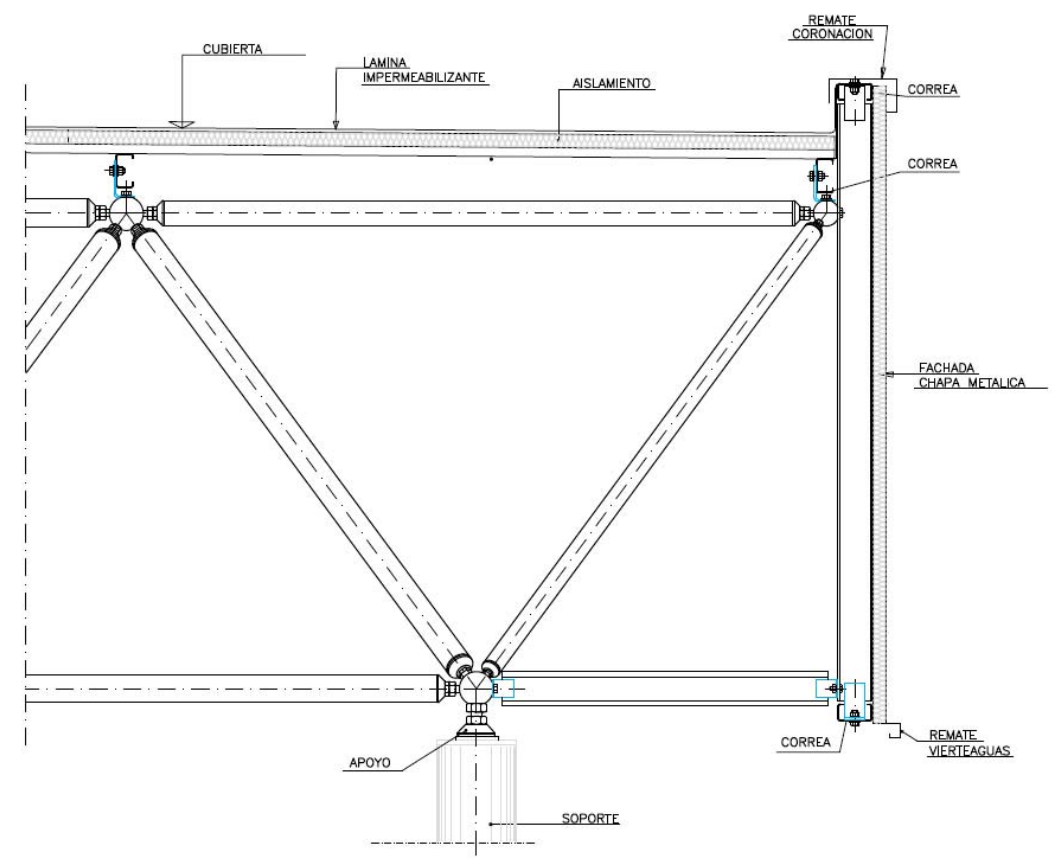

Figura 7. [Materialización] 


\section{FICHA TÉCNICA DE LA OBRA}

NOMBRE DEL PROYECTO: Terminal de Ómnibus de las Breñas (Chaco). Anexos: Oficina de Tránsito, Bromatología, Turismo y Cultura del Municipio de Las Breñas./ AUTORES: Iris Carolina Almanza; María del Huerto Martínez Quiroga; Gonzalo Cabás; Albano Mucchielli.

\section{INSTITUCIONES INVOLUCRADAS}

ORGANIZADOR: Sociedad de Arquitectos del Chaco.

PROMOTOR: Municipalidad de la Ciudad de Las Breñas. Departamento 9 de Julio, Provincia del Chaco./ FECHA DE PROYECTO: 18 de junio de 2014./ ÁREA DE TERRENO: $25.040 \mathrm{~m}^{2}$. 